Vol. 6(6), pp. 488-494, J une 2014

DOI: $10.5897 /$ J BC 2014.0698

Artic le Number: FBD477B45315

ISSN 2141-243X

Copyright (๑) 2014

Author(s) reta in the copyright of this artic le

International Journal of Biodiversity

http://www.academic joumals.org/lJ BC

\title{
Distribution of soil types, vegetation and tree species diversity in Eastern Ghats of Srikakulam District, Andhra Pradesh, India
}

\author{
Srinivasa Rao D.*, Prayaga Murthy P. and Aniel Kumar O. \\ Department of Botany, Andhra University, Visakhapatnam-530003, Andhra Pradesh, India.
}

Received 23 February 2014; Accepted 28 March 2014

\begin{abstract}
The present investigation was carried out on distribution of soil types, vegetation and tree species diversity in Eastern Ghats of Srikakulam District, Andhra Pradesh, India. The inventory of tree species was done in $\mathbf{4 0}$ different forest areas of Srikakulam district. All the sample plots are tropical and moist thorny forest and dry thorny scrub forests of Srikakulam district. The soils of the study area are compressed red soils, loamy soils, sandy loams, with varying proportions of sand and clay and it constitute $96 \%$ of the total area; red sandy soil is the common type. Tree species richness varied according to the disturbance gradient in the different stands, a total of 4744 individuals, belonging to 129 species, 96 genera among 46 families from 40 line transects were recorded in the study area. Species richness ranging from 47 to 9 in a transect was recorded in the present study. Highest species richness of sizes 47 for $65-\mathrm{N} / 14$ (SW-3, 65N-14 NW-3) was seen at Haddubanghi and lowest diversity 9 was seen at Korasanda 74-B/1(SE-1).
\end{abstract}

Key words: Soil types, vegetation, tree species diversity, Srikakulam district.

\section{INTRODUCTION}

Biodiversity is used in describing the diversity of life on earth, it includes all life forms and the ecosystem of which they are part. In the developing countries, biodiversity provides the assurance of food, many raw materials such as fibre for clothing, materials for shelter, fertilizers, fuel and medicines as well as sources of work energy in the form of animal traction. In addition, biodiversity maintains balance for planetary and human survival (Jafferies, 1997). Species diversity in the tropics varies dramatically from place to place, as compared to other tropical forest types, (Holdridge 1967). Dry deciduous forests are among the most exploited and endangered ecosystems of the biosphere (Murphy and Lugo, 1986; Gentry, 1992). Studies from forest survey of India showed that an average of $54 \%$ of forest is effected by fire and $72 \%$ of forest area is subjected to grazing annually, 3.73 million hectors of forest area are burnt resulting in economic losses of approximately 440 crores (MOE,1999). Dry deciduous forests are among the most exploited and endangered ecosystems of the biosphere (Janzen, 1988; Gentry, 1992). The world wide destruction of the natural environment by population explosion, urbanization,

*Corresponding author. E-mail: srinivas.duvvada@gmail.com.

Author(s) agree that this article remain permanently open access under the terms of the Creative Commons Attribution License 4.0 International License 
industrialization and habitat fragmentation has led to a tremendous loss of biological diversity over the past few decades. Over exploitation is to severely reduce the population sizes below the critical level and consequently the survival of the species. Phyto-sociological investigation of vegetation serves as a pre-requisite for investigating the details of the primary productivity of an ecosystem. Tree species diversity, distribution and population structure of tropical forests of Eastern Ghats are poorly understood. We analyzed the structure of tropical deciduous forests in Srikakulam district of Eastern Ghats, Andhra Pradesh, India.

\section{MATERIALS AND METHODS}

Phytosociological studies carried out during July 2008 to June 2011 covered all spectrum of vegetation. The entire stretches of Eastern Ghats of Srikakulam district are divided into $6.25 \times 6.25 \mathrm{~km}$ grid, based on the toposheets obtained. This expertise method of classification is obtained from UAS-ATREE team Bangalore (Jagadish et al., 2003). Each grid is from a sampling unit. The inventory of tree species was done in 40 different forest areas in Srikakulam district. In each forest area consider as one belt transect, one belt transect (Plot) of $5 \times 1000 \mathrm{~m}$ in each of the $6.25 \mathrm{x}$ $6.25 \mathrm{~km}$ (grid) is a sampling protocol with $0.01 \%$ of sampling intensity based on random sampling method. All the plots samples are from tropical dry deciduous forests, moist deciduous forests and scrub deciduous forests of Srikakulam district. In order to revisit these plots for seasonal sampling, latitude, longitude, altitude values were recorded by using a GPS (Garmen India) and other Geo-climatic features were identified and represented in Tables 1 and 2. The specimens were identified with the help of flora of Andhra Pradesh 3 Volumes (Pullaiah et al., 1997) and local floras like Flora of Srikakulam district (Rao R.S. and Hara Sreeramulu, 1986), studies on the vegetation and flora of Vizianagaram district (Venkaiah, 1980) and (Srinivasa et al., 2012) phytosociological studies on tree diversity of Srikakulam districts of Andhra Pradesh, India.

\section{Study area}

The Srikakulam district lies on the east coast of India between $18^{\circ}$ $20^{\prime}$ and $19^{\circ}-10^{\prime} \mathrm{N}$ and $83^{\circ}-50^{\prime}$ and $84^{\circ}-50^{\prime} \mathrm{E}$. The total geographical area is $2,254 \mathrm{sq} \mathrm{km}$. This area is bounded by Orissa State on the North and West and Bay of Bengal on the East and North east on the south and west Visakhapatnam district. These area consist of 37 mandals of different types of soils like red soils, loamy soils, sandy loams, with varying proportions of sand and clay and they constitute $96 \%$ of the total area .Red sandy soils area the common type. The climate of the region is generally tropical. The temperature in the hill areas is cooler than in plains because hills receive heavier rainfall. The mean maximum temperature is $30-40^{\circ} \mathrm{C}$ in April-May and the mean minimum temperature is $17.4^{\circ} \mathrm{C}$ in December-January during the summer season till the on-set of the South-West monsoon the heat is oppressive and the day temperature may sometimes be about $43^{\circ} \mathrm{C}$. The rainfall in the region is considerably more in the hilly areas as compared to the plains.

\section{RESULTS AND DISCUSSION}

A total of 129 tree species were recorded from 40 tran- sects; the predominant species of this region are Mangifera indica which is the most important species followed by Lannea coromandelica, Wrightia tinctoria, Dalbergia paniculata, Tamarindus indica, Diospyros sylvatica, Cleistanthus collinus, Xylia xylocarpa, Chloroxylon swietenia and Terminalia alata were recorded. Density, frequency, relative density, relative frequency and relative abundance values were taken for the preparation of single link cluster analysis and results revealed that the majority of the species formed similar groups (Scale 0-25) except M. indica and T.S indica which forms the dissimilar groups in the study area (Figure 1). Latitude, longitude and altitude readings of the sampling areas and different soil types like sandy, black, red, loam and with different combinations were recorded and presented in Table 1. In the present investigation, the highest altitude was recorded in Laada followed by Polla, Kothakota, Samarillu, Sara, Sunnapugedda and lowest altitude was recorded in Sakipuram. Total study area was divided into 40 grids, the number of species that occurred in each grid was recorded; 47 species in Huddubanghi (65N-14,SW-3) followed by 45 species in near Chapara (74B-1 SE-4) and Sundarada (74B-2 NE-1), 43 species at Sobha (74B-1(SW-2), 42 species at Sunnapagedda (65N-10 NE-3), 41 species at Yetugada (65 N-13 NW-2) etc were recorded in the entire forest area (Table 2). Tree species richness varied according to disturbance gradient in different, a total of 4744 individuals, belonging to 129 species, 96 genera among 46 families from 40 line transects in the study area are recorded in Tables 1 and 2). Species richness ranges from 47 to 9 in a transect, as recorded in the present study. Species richness was more 47 for $65-\mathrm{N} / 14(\mathrm{SW}-3,65 \mathrm{~N} / 14 \mathrm{NW}-3$ ) at Haddubanghi and has least species diversity 9 at Korasanda $74 \mathrm{~B} / 1$ (SE-1). Species area and species individual accumulation curve against equal-sized sampling area in different vegetation types showed that species heterogeneity was higher in vegetation types at mid elevations while their abundance was higher in vegetation types at higher elevations (Jayakumar and Nair, 2013). In tropical rain forests, the ranges of tree species count per hectare is about 20 to maximum of 223 (Parthasarathy and Sethi, 1997), 42-47 species ha ${ }^{-1}$ (Kadavul and Parthasarathy, 1998). In the present investigations, maximum of 47 tree species per $1000 \mathrm{~m}$ (one transect) was recorded, these results agree with earlier observations of Parthasarathy and Sethi (1997) and Kadavul and Parthasarathy (1998). In the present study, species richness in study sites are also correlated with the taxonomical studies, most of the trees show random distribution and was lowwhen compared with that of tropical forests of Indian Eastern Ghats and Western Ghats, that is, the number of species in Nallamalias, 69 (Sudhakar et al., 2008), Kolli hills, 2556 (Chittibabu and Parthasaratthy, 2000), Kalarayan hills, 42-47 (Kadavul and Parthasarathy, 1999a). Shervarayan hills, 33-50 (Kadavul and Parthasarathy, 1999 b). The sacred grooves of Kerala 14-23, (Chandra Sekhar and 
Table 1. Latitude, longitude and altitude of the sampling areas and soil types of the study area.

\begin{tabular}{|c|c|c|c|c|c|c|c|}
\hline \multirow[b]{2}{*}{ Location } & \multicolumn{2}{|c|}{ Latitude } & \multicolumn{2}{|c|}{ Longitude } & \multicolumn{2}{|l|}{ Altitude } & \multirow[b]{2}{*}{ Soil type } \\
\hline & Starting & Ending & Starting & Ending & $\begin{array}{l}\text { Starting } \\
\text { (mts) }\end{array}$ & $\begin{array}{l}\text { Ending } \\
\text { (mts) }\end{array}$ & \\
\hline Akkarajupeta & $18^{\circ} 43^{\prime} 223^{\prime \prime} N$ & $18^{\circ} 43^{\prime} 672^{\prime \prime} \mathrm{N}$ & $83^{\circ} 39^{\prime} 523^{\prime \prime} \mathrm{E}$ & $83^{\circ} 39^{\prime} 848 " \mathrm{E}$ & 74 & 186 & Black \\
\hline Bethalapuram & $18^{\circ} 58^{\prime} 923^{\prime \prime} \mathrm{N}$ & $18^{\circ} 59^{\prime} 234^{\prime \prime} \mathrm{N}$ & $84^{\circ} 3^{\circ}$ ' $31^{\prime \prime} \mathrm{E}$ & $84^{\circ} 3^{\circ} 325^{\prime \prime} \mathrm{E}$ & 86 & 121 & Red \\
\hline Bharani kota & $18^{\circ} 17^{\prime} 479^{\prime \prime} \mathrm{N}$ & $18^{\circ} 17^{\prime} 731^{\prime \prime} \mathrm{N}$ & $84^{\circ} 47^{\prime} 2^{\circ} 1^{\prime \prime} \mathrm{E}$ & $84^{\circ} 47^{\prime} 429^{\prime \prime} \mathrm{E}$ & 35 & 86 & Red \\
\hline Degalakotturu & $18^{\circ} 43^{\prime} 422^{\prime \prime} N$ & $18^{\circ} 43^{\prime} 631^{\prime \prime} N$ & $84^{\circ} 42^{\prime} 7^{\circ} 2^{\prime \prime} \mathrm{E}$ & $84^{\circ} 42^{\prime} 873$ 'E & 78 & 89 & Red \\
\hline Haddubangi & $18^{\circ} 45^{\prime} 326^{\prime \prime} \mathrm{N}$ & $18^{\circ} 45^{\prime} 683^{\prime \prime} \mathrm{N}$ & $83^{\circ} 42^{\prime} 231^{\prime \prime} \mathrm{E}$ & $83^{\circ} 42^{\prime} 62^{\circ} " \mathrm{E}$ & 116 & 214 & Red \\
\hline Hannali & $18^{\circ} 53^{\prime} 942$ "N & $18^{\circ} 54^{\prime} 1^{\circ} 3 " N$ & $84^{\circ} 28$ ' 834" E & $84^{\circ} 28^{\prime} 625$ "E & 35 & 86 & Red \\
\hline Haripuram & $18^{\circ} 35^{\prime} 832^{\prime \prime} \mathrm{N}$ & $18^{\circ} 35^{\prime} 643^{\prime \prime} \mathrm{N}$ & $83^{\circ} 47^{\prime} 389^{\prime \prime} E$ & $83^{\circ} 47^{\prime} 526^{\prime \prime} \mathrm{E}$ & 58 & 110 & Red \\
\hline Hussanpuram & $18^{\circ} 4^{\circ} 279$ "N & $18^{\circ} 4^{\circ} 636^{\prime \prime} \mathrm{N}$ & $83^{\circ} 39^{\prime} 8^{\circ} 3^{\prime \prime} \mathrm{E}$ & $83^{\circ} 39^{\prime} 917^{\prime \prime} \mathrm{E}$ & 78 & 96 & Red \\
\hline Irupeduguda & $18^{\circ} 42^{\prime} 33^{\circ}$ 'N & $18^{\circ} 42^{\prime} 656^{\prime \prime} \mathrm{N}$ & $83^{\circ} 53^{\prime} 175^{\prime \prime} \mathrm{E}$ & $83^{\circ} 53^{\prime} 268$ "E & 327 & 346 & Black \\
\hline Kalandinagaram & $18^{\circ} 56^{\prime} 826$ "N & $18^{\circ} 56^{\prime} 798$ "N & $84^{\circ} 26^{\prime} 511^{\prime \prime} \mathrm{E}$ & $84^{\circ} 25^{\prime} 838^{\prime \prime} \mathrm{E}$ & 71 & 180 & Black \\
\hline Karakavalasa & $18^{\circ} 35^{\prime \circ}{ }^{\circ} " \mathrm{~N}$ & $18^{\circ} 34^{\prime} 733$ '” & $83^{\circ} 54^{\prime} 762 " \mathrm{E}$ & $83^{\circ} 54$ ' 464" E & 53 & 80 & Black \\
\hline Korasanda & $18^{\circ} 49^{\prime} 782$ "N & $18^{\circ} 49^{\prime} 512^{\prime \prime} \mathrm{N}$ & $84^{\circ} 1^{\circ \prime} 226^{\prime \prime} \mathrm{E}$ & $84^{\circ} 1^{\circ} 489^{\prime \prime} \mathrm{E}$ & 72 & 89 & Red \\
\hline Kothakota & $18^{\circ} 44^{\prime} 154$ 'N & $18^{\circ} 44^{\prime} 186^{\prime \prime} \mathrm{N}$ & $83^{\circ} 39^{\prime} 27^{\circ \prime} \mathrm{E}$ & $83^{\circ} 39$ ' 776 'E & 504 & 499 & Red \\
\hline Laada & $18^{\circ} 45^{\prime} 233^{\prime \prime} \mathrm{N}$ & $18^{\circ} 45^{\prime} 372^{\prime \prime} \mathrm{N}$ & $83^{\circ} 45^{\prime} 434^{\prime \prime} \mathrm{E}$ & $83^{\circ} 45^{\prime} 543^{\prime \prime} \mathrm{E}$ & 667 & 739 & Red \\
\hline Labba & $18^{\circ} 43^{\prime} 586$ "N & $18^{\circ} 43^{\prime} 759$ 'N & $83^{\circ} 52^{\prime} 943^{\prime \prime} \mathrm{E}$ & $83^{\circ} 52^{\prime} 625^{\prime \prime} \mathrm{E}$ & 125 & 142 & Red \\
\hline Machannapeta & $18^{\circ} 32^{\prime} 4^{\circ} 9 " \mathrm{~N}$ & $18^{\circ} 32^{\prime} 725^{\prime \prime} \mathrm{N}$ & $83^{\circ} 51^{\prime} 969 " \mathrm{E}$ & $83^{\circ} 51^{\prime} 834^{\prime \prime} \mathrm{E}$ & 101 & 199 & Red \\
\hline Manumakonda & $18^{\circ} 59^{\prime} 423^{\prime \prime} \mathrm{N}$ & $18^{\circ} 59^{\prime} \circ 15^{\prime \prime} \mathrm{N}$ & $83^{\circ} 46^{\prime} 4^{\circ} 5^{\prime \prime} \mathrm{E}$ & $83^{\circ} 45^{\prime} 997 ' \mathrm{E}$ & 108 & 186 & Red \\
\hline Masimguda & $18^{\circ} 45^{\prime} 883^{\prime \prime} \mathrm{N}$ & $18^{\circ} 45^{\prime} 7^{\circ} 9$ "'N & $83^{\circ} 51^{\prime} 75^{\circ \prime} \mathrm{E}$ & $83^{\circ} 51^{\prime} 791^{\prime \prime} \mathrm{E}$ & 125 & 169 & Silt Red \\
\hline Mukundapuram & $18^{\circ} 44^{\prime} 152^{\prime \prime} \mathrm{N}$ & $18^{\circ} 43^{\prime} 756$ "N & $83^{\circ} 51^{\prime} 712^{\prime \prime} \mathrm{E}$ & $83^{\circ} 51^{\prime} 3^{\circ} 4^{\prime \prime} \mathrm{E}$ & 116 & 214 & Red \\
\hline Nallarayiguda & $18^{\circ} 51^{\prime}{ }^{\circ} 73^{\prime \prime} \mathrm{N}$ & $18^{\circ} 51^{\prime} 24^{\circ} " \mathrm{~N}$ & $83^{\circ} 5^{\circ} 192 " \mathrm{E}$ & $83^{\circ} 5^{\circ} 614^{\prime \prime} \mathrm{E}$ & 196 & 69 & Black \\
\hline Nearchepara & $18^{\circ} 47^{\prime} 782^{\prime \prime} \mathrm{N}$ & $18^{\circ} 47^{\prime} 435^{\prime \prime} \mathrm{N}$ & $84^{\circ} 14^{\prime} 2^{\circ} 1^{\prime \prime} \mathrm{E}$ & $84^{\circ} 14^{\prime} 535^{\prime \prime} \mathrm{E}$ & 55 & 63 & Red \\
\hline Pasukudi & $18^{\circ} 55^{\prime} 628^{\prime \prime} \mathrm{N}$ & $18^{\circ} 55^{\prime} 182^{\prime \prime} \mathrm{N}$ & $83^{\circ} 49^{\prime \circ} 9^{\circ} \mathrm{E}$ & $83^{\circ} 48$ ' 922" E & 89 & 71 & Red \\
\hline Peddakedari & $18^{\circ} 39^{\prime} 47^{\circ} " \mathrm{~N}$ & $18^{\circ} 39^{\prime} 642^{\prime \prime} \mathrm{N}$ & $84^{\circ} 13^{\prime} 221^{\prime \prime} E$ & $84^{\circ} 13^{\prime} 535^{\prime \prime} \mathrm{E}$ & 47 & 89 & Red \\
\hline Peddasankili & $18^{\circ} 42^{\prime} 287^{\prime \prime} \mathrm{N}$ & $18^{\circ} 42^{\prime} 556^{\prime \prime} \mathrm{N}$ & $83^{\circ} 55^{\prime} 693$ "'E & $83^{\circ} 55^{\prime} 949^{\prime \prime} \mathrm{E}$ & 61 & 109 & Red \\
\hline Polla & $18^{\circ} 45^{\prime} 782$ "N & $18^{\circ} 45^{\prime} 323^{\prime \prime} N$ & $83^{\circ} 41^{\prime} 198 ” E$ & $83^{\circ} 41^{\prime} 71^{\circ \prime} \mathrm{E}$ & 503 & 487 & Black \\
\hline Rayala & $18^{\circ} 48^{\prime}{ }^{\circ} 58^{\prime \prime} \mathrm{N}$ & $18^{\circ} 47^{\prime} 823^{\prime \prime} \mathrm{N}$ & $83^{\circ} 59^{\prime}{ }^{\circ} 92$ 'E & $83^{\circ} 59^{\prime} 457^{\prime \prime} \mathrm{E}$ & 69 & 193 & Red \\
\hline S.Narsipatnam & $18^{\circ} 44^{\prime} 689^{\prime \prime} \mathrm{N}$ & $18^{\circ} 44^{\prime} 987^{\prime \prime} \mathrm{N}$ & $83^{\circ} 36^{\prime} 446^{\prime \prime} \mathrm{E}$ & $83^{\circ} 36^{\prime} 223^{\prime \prime} \mathrm{E}$ & 93 & 106 & Black \\
\hline Sakipuram & $18^{\circ} 45^{\prime} 793^{\prime \prime} \mathrm{N}$ & $18^{\circ} 45^{\prime} 932 " \mathrm{~N}$ & $84^{\circ} 2^{\circ}$ ' $556^{\prime \prime} \mathrm{E}$ & $84^{\circ} 2^{\circ} 937^{\prime \prime} \mathrm{E}$ & 31 & 49 & Red \\
\hline Samarillu & $18^{\circ} 43^{\prime} 77^{\circ} " \mathrm{~N}$ & $18^{\circ} 43^{\prime} 673^{\prime \prime} \mathrm{N}$ & $83^{\circ} 45^{\prime} \circ 15^{\prime \prime} \mathrm{E}$ & $83^{\circ} 45^{\prime} 387^{\prime \prime} E$ & 475 & 484 & Loam \\
\hline Sambam & $18^{\circ} 38^{\prime} 7^{\circ} 3$ "N & $18^{\circ} 38^{\prime} 171^{\prime \prime} \mathrm{N}$ & $83^{\circ} 5^{\circ} 975^{\prime \prime} \mathrm{E}$ & $83^{\circ} 51^{\prime} 235^{\prime \prime} \mathrm{E}$ & 310 & 287 & Silt black \\
\hline Samparai guda & $18^{\circ} 45^{\prime} 384^{\prime \prime} \mathrm{N}$ & $18^{\circ} 45^{\prime} 789$ "N & $83^{\circ} 52^{\prime} 262 " \mathrm{E}$ & $83^{\circ} 52^{\prime} 638$ 'E & 89 & 71 & Red \\
\hline Sara & $18^{\circ} 35^{\prime} 6^{\circ} 6^{\prime \prime} \mathrm{N}$ & $18^{\circ} 35^{\prime} 577^{\prime \prime} \mathrm{N}$ & $83^{\circ} 5^{\circ} 645^{\prime \prime} \mathrm{E}$ & $83^{\circ} 49^{\prime} 785^{\prime \prime E}$ & 353 & 372 & Red \\
\hline Saribujjili & $18^{\circ} 32^{\prime}{ }^{\circ} 12^{\prime \prime} \mathrm{N}$ & $18^{\circ} 32^{\prime} 619^{\prime \prime N}$ & $83^{\circ} 54 ' 597^{\prime \prime} \mathrm{E}$ & $83^{\circ} 54^{\prime} 444$ 'E & 39 & 174 & Red \\
\hline Soba & $18^{\circ} 47^{\prime} 436^{\prime \prime} \mathrm{N}$ & $18^{\circ} 47^{\prime} 6^{\circ} 8^{\prime \prime} \mathrm{N}$ & $84^{\circ} \circ 1$ ' 836" E & $84^{\circ}{ }^{\circ} 1^{\prime} 52^{\circ} " \mathrm{E}$ & 86 & 110 & Red \\
\hline Sundarada & $18^{\circ} 44^{\prime} 567^{\prime \prime} \mathrm{N}$ & $18^{\circ} 44^{\prime} 998$ "N & $84^{\circ}{ }^{\circ} 8$ ' $634^{\prime \prime E}$ & $84^{\circ \circ} 8$ ' 935 "'E & 80 & 124 & Red \\
\hline Sunnapugedda & $18^{\circ} 45^{\prime} 624^{\prime \prime} \mathrm{N}$ & $18^{\circ} 46^{\prime} 128^{\prime \prime} \mathrm{N}$ & $83^{\circ} 42^{\prime} 449^{\prime \prime} \mathrm{E}$ & $83^{\circ} 42^{\prime} 419^{\prime \prime} \mathrm{E}$ & 325 & 336 & Black \\
\hline Temburu & $18^{\circ} 37^{\prime} 538^{\prime \prime} \mathrm{N}$ & $18^{\circ} 37^{\prime} 84^{\circ} " \mathrm{~N}$ & $84^{\circ} \circ 7^{\prime} 678^{\prime \prime} \mathrm{E}$ & $84^{\circ} \circ 7^{\prime} 937^{\prime \prime} \mathrm{E}$ & 59 & 98 & Red \\
\hline Timedisala & $18^{\circ} 43^{\prime} 325^{\prime \prime} \mathrm{N}$ & $18^{\circ} 43^{\prime} 712^{\prime \prime} \mathrm{N}$ & $84^{\circ} 18$ ' 235" E & $84^{\circ} 18^{\prime} 612^{\prime \prime} \mathrm{E}$ & 56 & 74 & Red \\
\hline Vampaliguda & $18^{\circ} 44^{\prime} 979$ "N & $18^{\circ} 45^{\prime} 1^{\circ} 7^{\prime \prime} \mathrm{N}$ & $83^{\circ} 51^{\prime} 356^{\prime \prime} \mathrm{E}$ & $83^{\circ} 51^{\prime} 479$ "'E & 105 & 186 & Red \\
\hline Yatuguda & $18^{\circ} 53^{\prime} 658^{\prime \prime} \mathrm{N}$ & $18^{\circ} 53$ '655" N & $83^{\circ} 47^{\prime} 761^{\prime \prime} \mathrm{E}$ & $83^{\circ} 47^{\prime} 264$ " E & 151 & 331 & Black \\
\hline
\end{tabular}

Sankar, 1998), Thirumani Kuzhi sacred groove, 38 (Parthasarathy and Karthikeyan, 1997). The predominant forest areas of the study regions of Srikakulam district in Andhra Pradesh are tropical deciduous forests (Champion and Seth, 1968). Studies in this area reveal that the most abundant families were Rubiaceae and Mimosaceae (13), Moraceae (12), Euphorbiaceae (11),
Fabaceae (9), Verbenaceae (9), Rutaceae, Anacardiaceae, Combretaceae and Ebenaceae with 6 species, respectively. An obvious variation in representation of tree species and the proportion of dominant species in the forests can directly be attributed to rainfall distribution and favorable topographic conditions. The present study also support the above fact that Euphorbiaceae, Fabaceae 
Table 2. Details of the study area.

\begin{tabular}{|c|c|c|c|c|c|c|c|c|c|}
\hline $\begin{array}{l}\text { Toposheet } \\
\text { number }\end{array}$ & $\begin{array}{l}\text { Grid } \\
\text { number }\end{array}$ & Location & District & $\begin{array}{l}\text { Forest } \\
\text { Division } \\
\end{array}$ & $\begin{array}{l}\text { Forest } \\
\text { range }\end{array}$ & $\begin{array}{l}\text { Type of } \\
\text { vegetation }\end{array}$ & $\begin{array}{l}\text { Families } \\
\text { number }\end{array}$ & $\begin{array}{l}\text { Genera } \\
\text { number }\end{array}$ & $\begin{array}{l}\text { Species } \\
\text { number }\end{array}$ \\
\hline $65 N-10$ & NE-4 & Akkarajupeta & Srikakulam & Srikakulam & Palakonda & $\mathrm{DD}$ & 18 & 31 & 35 \\
\hline 74 B-9 & NW-1 & Bethalapuram & Srikakulam & Srikakulam & Pathapatnam & SCRUB & 21 & 29 & 34 \\
\hline 74 B-5 & NE-2 & Bharanikota & Srikakulam & Srikakulam & Srikakulam & SCRUB & 20 & 22 & 25 \\
\hline 74 B-2 & NE-3 & Degalakotturu & Srikakulam & Srikakulam & Pathapatnam & SCRUB & 11 & 12 & 12 \\
\hline $65 \mathrm{~N}-14$ & SW-3 & Haddubangi & Srikakulam & Srikakulam & Pathapatnam & SCRUB & 30 & 43 & 47 \\
\hline 74 B-5 & NE-4 & Hannali & Srikakulam & Srikakulam & Srikakulam & SCRUB & 20 & 22 & 25 \\
\hline $65 \mathrm{~N}-14$ & SW-1 & Haripuram & Srikakulam & Srikakulam & Palakonda & SCRUB & 23 & 30 & 33 \\
\hline $65 N-10$ & $\mathrm{NE}-2$ & Hussanpuram & Srikakulam & Srikakulam & Palakonda & SCRUB & 17 & 21 & 22 \\
\hline $65 \mathrm{~N}-14$ & $N E-1$ & Irupeduguda & Srikakulam & Srikakulam & Palakonda & D D & 18 & 23 & 23 \\
\hline 74B-5 & $N E-3$ & KalandiNagaram & Srikakulam & Srikakulam & Pathapatnam & $\mathrm{DD}$ & 22 & 31 & 33 \\
\hline $65 \mathrm{~N}-14$ & SE-1 & Karakavalasa & Srikakulam & Srikakulam & Pathapatnam & SCRUB & 21 & 32 & 36 \\
\hline 74B-1 & SE-1 & Korasanda & Srikakulam & Srikakulam & Pathapatnam & SCRUB & 9 & 9 & 9 \\
\hline $65 N-10$ & NE-1 & Kothakota & Srikakulam & Srikakulam & Palakonda & $\mathrm{MD}$ & 23 & 34 & 38 \\
\hline $65 N-13$ & SW-2 & Laada & Srikakulam & Srikakulam & Palakonda & $\mathrm{DD}$ & 21 & 30 & 33 \\
\hline $65 \mathrm{~N}-14$ & NE-1 & Labba & Srikakulam & Srikakulam & Palakonda & D D & 20 & 26 & 26 \\
\hline $65 \mathrm{~N}-14$ & SW-4 & Machanna peta & Srikakulam & Srikakulam & Palakonda & DD & 24 & 33 & 37 \\
\hline $65 \mathrm{~N}-13$ & NW-1 & Manumakonda & Srikakulam & Srikakulam & Palakonda & DD & 11 & 13 & 14 \\
\hline $65 \mathrm{~N}-13$ & SW-4 & Masimguda & Srikakulam & Srikakulam & Palakonda & DD & 21 & 31 & 32 \\
\hline $65 \mathrm{~N}-14$ & NW-3 & Mukundapuram & Srikakulam & Srikakulam & Pathapatnam & SCRUB & 30 & 43 & 47 \\
\hline $65 N-13$ & SW-3 & Nallarayiguda & Srikakulam & Srikakulam & Palakonda & SCRUB & 20 & 27 & 33 \\
\hline 74 B-1 & SE-4 & Near Chapara & Srikakulam & Srikakulam & Pathapatnam & DD & 22 & 39 & 45 \\
\hline $65 N-13$ & NW-4 & Pasukudi & Srikakulam & Srikakulam & Palakonda & SCRUB & 21 & 27 & 30 \\
\hline 74B-2 & NE-4 & Peddakedari & Srikakulam & Srikakulam & Pathapatnam & $\mathrm{DD}$ & 22 & 33 & 38 \\
\hline $65 \mathrm{~N}-14$ & $N E-3$ & Peddasankili & Srikakulam & Srikakulam & Palakonda & D D & 18 & 23 & 23 \\
\hline $65 N-9$ & SE-4 & Polla & Srikakulam & Srikakulam & Palakonda & D D & 22 & 34 & 36 \\
\hline $65 N-13$ & SE-4 & Rayala & Srikakulam & Srikakulam & Pathapatnam & SCRUB & 21 & 26 & 28 \\
\hline $65 N-10$ & NW-3 & S.Narsipatnam & Srikakulam & Srikakulam & Palakonda & SCRUB & 15 & 16 & 18 \\
\hline 74 B-5 & SW-4 & Sakipuram & Srikakulam & Srikakulam & Srikakulam & SCRUB & 11 & 12 & 14 \\
\hline $65 \mathrm{~N}-14$ & NW-1 & Samarillu & Srikakulam & Srikakulam & Palakonda & DD & 24 & 36 & 39 \\
\hline $65 \mathrm{~N}-14$ & NW-4 & Sambam & Srikakulam & Srikakulam & Palakonda & DD & 21 & 35 & 38 \\
\hline $65 \mathrm{~N}-13$ & SW-1 & Samparai guda & Srikakulam & Srikakulam & Palakonda & SCRUB & 21 & 27 & 30 \\
\hline $65 \mathrm{~N}-14$ & NE-2 & Sara & Srikakulam & Srikakulam & Palakonda & $\mathrm{DD}$ & 21 & 32 & 33 \\
\hline $65 N-14$ & SE-2 & Saribujjili & Srikakulam & Srikakulam & Pathapatnam & SCRUB & 11 & 13 & 14 \\
\hline 74B-1 & SW-2 & Soba & Srikakulam & Srikakulam & Pathapatnam & DD & 22 & 35 & 43 \\
\hline 74B-2 & NE-1 & Sundarada & Srikakulam & Pathapatnam & Srikakulam & SCRUB & 30 & 41 & 45 \\
\hline $65 N-10$ & $N E-3$ & Sunnapugedda & Srikakulam & Srikakulam & Palakonda & $\mathrm{DD}$ & 25 & 38 & 42 \\
\hline 74B-2 & NE-2 & Temburu & Srikakulam & Srikakulam & Srikakulam & SCRUB & 24 & 35 & 39 \\
\hline $74 B-6$ & NW-1 & Timedisala & Srikakulam & Srikakulam & Pathapatnam & SCRUB & 23 & 32 & 37 \\
\hline $65 \mathrm{~N}-14$ & NW-3 & Vampaliguda & Srikakulam & Srikakulam & Palakonda & DD & 22 & 32 & 32 \\
\hline $65 N-13$ & NW-2 & Yetuguda & Srikakulam & Srikakulam & Palakonda & DD & 23 & 37 & 41 \\
\hline
\end{tabular}

and Rubiaceae are dominant families in almost all type of forests as reported by Sudhakar et al. (2008) and Kadavul and Parthasarathy (1999a). In moist deciduous forests, the species composition comprise a mixture of both moist and dry elements; indicating transitional zone. In this forest, Anogeissus latifolia, Garuga pinnata, Haldinia cordifolia, Lagerstroemia parviflora, Lannea coromandelica, Mangifera indica, Protium serratum,
Pterocarpus marsupium, Syzygium cuminii, Terminalia alata and Xylia xylocarpa are predominant species, some tree species like Chloroxylon swietenia, Diospyros sylvatica, Schleichera oleosa, etc. grow luxuriantly reaching more than $15-20 \mathrm{~m}$ in height. In dry deciduous forests, Bombax ceiba, Bridelia retusa, Dalbergia paniculata, Gmelina arborea, Mitragyna parvifolia, Sterculia urens, Strychnos nux-vomica, Terminalia alata, 


\section{Dendrogram using Single Linkage}

Rescaled Distance Cluster Combine

C A S E

Label

Vitex negunda

Vitex pinnata

Acacia melanoxylon

Callicarpa arborea

Vitex altissima

Gardenia gummifera

Holarrhena pubescens

Macaranga peltata

Phoenix sylvestris

Alstonia venenata

Strychnos potatorum

Albizia amara

Holoptelea integrifolia

Ficus benghalensis

Ceiba pentandra

Ficus tomentosa

Pavetta tomentosa

Pavetta indica

Murraya paniculata

Trema orientalis

Ficus microcarpa

Miliousa tomentosa

Erythrina variegata

Oroxylum indicum

Firmiana colarata

Sapindus emarginatus

Terminalia arjuna

Terminalia chebula

Wrightia arborea

Gmelina arborea

Hymenodictyon orixense

Syzygium cumini

Erythrina suberosa

ochna obtusata

Ailanthus excelsa

Ficus semicordata

Ficus racemosa

Polyalthia suberosa

Maytenus emarginata

Antidesma ghaesambella

Cordia dichotoma

Litsea deccanensis

Soymida febrifuga

Albizia chinensis

Ziziphus mauritiana

Canthium parviflorum

Securinega virosa

Dichrostachys cinerea

Ficus mollis

Butea monosperma

Flacourtia jangomas

Borasassus flabellifer

Morinda pubescens

Artocarpus heterophyllus

Phyllanthus emblica

Kydia calycina

Dillenia pentagyna

Albizia odoratissima

Anogeissus acuminata

Nyctanthes arbortristis

Maba buxifolia

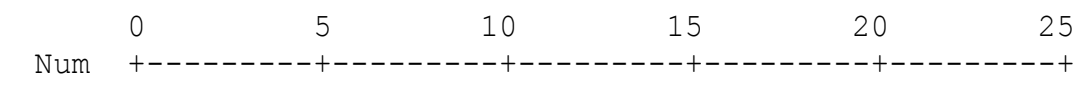

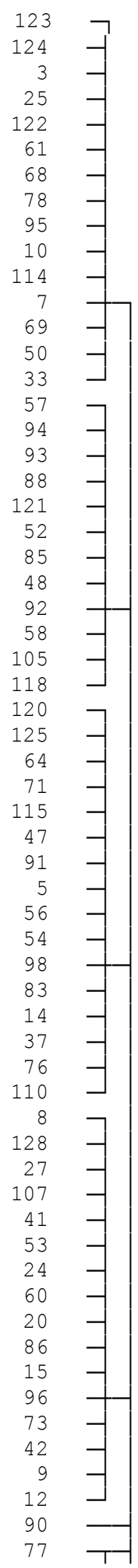

Figure 1. Hierarchical cluster analysis. 
Pongamia pinnata

Catunaregum spinosa

Erythroxylum monogynum

Annona squamosa

Ficus religiosa

Naringi crenulata

ziziphus xylopyrus

Hugonia mystax

Lagerstroemia parviflora

Polyalthia cerasoides

Manilkara hexandra

Madhuca longifolia

Atalantia monophylla

Grewia tiliaefolia

Shorea robusta

Dendrocalamus stricta

Flacourtia indica

Ixora arborea

Gardenia latifolia

Protium serratum

Ficus hispida

Careya arborea

Haldinia cordifolia

Diospyros chloroxylon

Mallotus philippensis

Garuga pinnata

Schleichera oleosa

Bombax ceiba

Diospyros montana

Cochlospermum religiosum

Memecylon edule

Streblus asper

Strychnos nux-vomica

Terminalia bellerica

Morinda tinctoria

Tamarindus indica

Mangifera indica

Chloroxylon swietenia

Lannea coromandelica

Anogeissus latifolia

Aegle marmelos

Terminalia alata

Cleistanthus collinus

Bauhinia racemosa

Acacia leucophloea

Alangium salvifolium

Xylia xylocarpa

Wrightia tinctoria

Sterculia urens

Cassia fistula

Premna latifolia

Dalbergia paniculata

Bridelia retusa

Premna tomentosa

Diospyros melanoxylon

Pterospermum xylocarpum

Bridelia montana

Buchanania lanzan

Azadirachta indica

Dalbergia latifolia

Gyrocarpus americanus

Pterocarpus marsupium

Canthium dicoccum

Casearia elliptica

Caryota urens

Diospyros sylvatica

Semecarpus anacardium

Acacia chundra

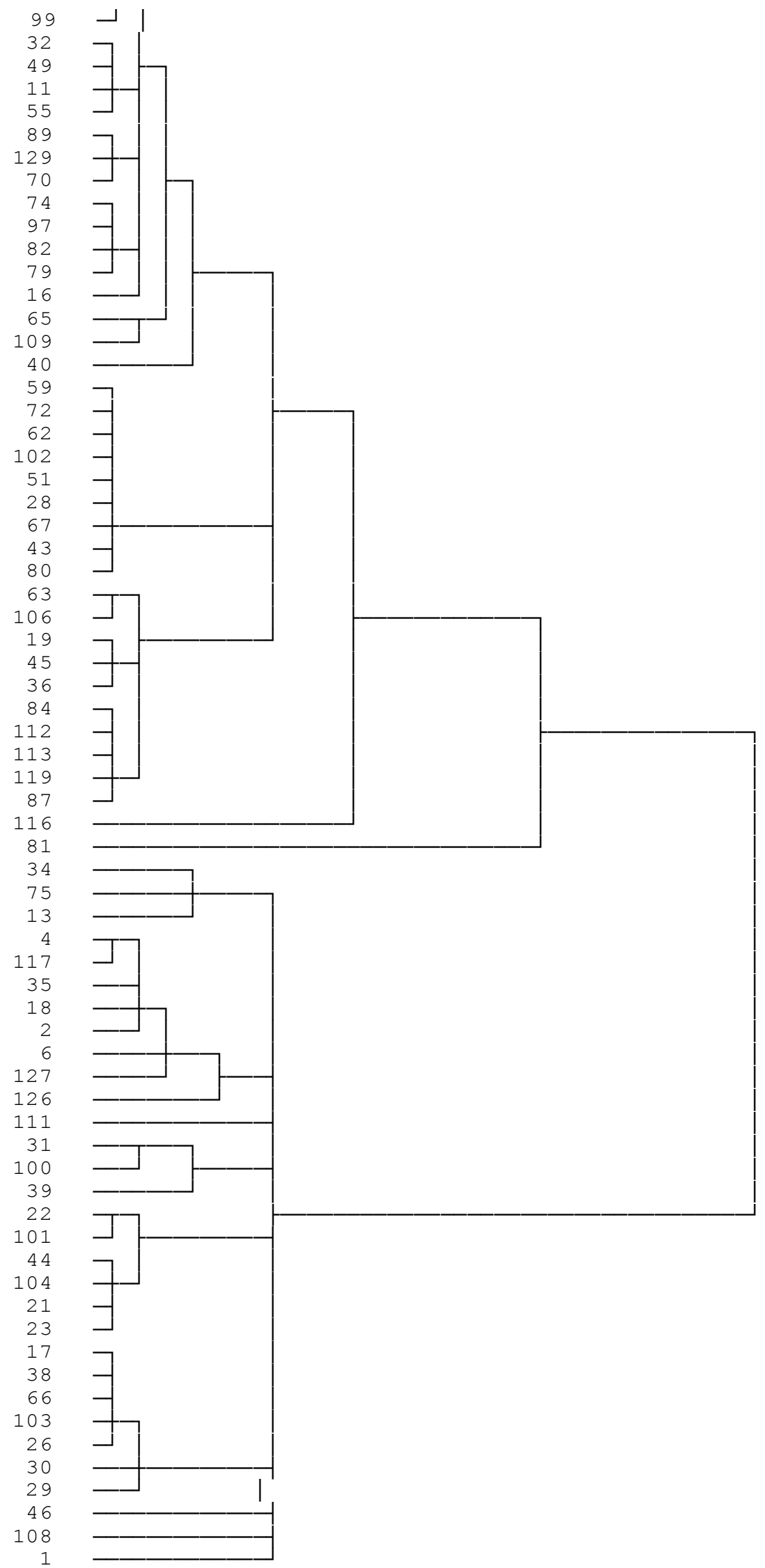

Figure 1 Contd. 
Terminalia bellerica and Xylia xylocarpa are predominant tree species found in red sandy loam soils. Scrub deciduous forest is represented by Diospyros melanoxylon, Diospyros chloroxylon, Strychnos potatorum, Zizyphus mauritiana, Wrightia tinctoria, Manilkara hexandra, Erythroxylum monogynum species were reported because soils are characterized by red sandy types in study area. Studies revealed the presence of 4744 individuals in Srikakulam district when compared with Nallamalias, Seshachalam and Nigidi hills (1541-3ha ${ }^{-1}$ ) (Sudhakara et al., 2008), Similipal Biosphere reserve (4819-8ha ${ }^{-1}$ ) Sudhakara et al., 2007), Boudh district, Orissa (2364-4 ha ${ }^{-1}$ ) (Sahu et al.,2007), inland and coastal tropical dry evergreen forest of Peninsular India

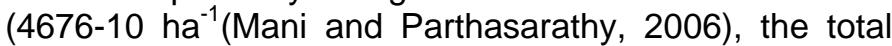
individuals reported in the study area is less when compared with various sites in Eastern Ghats, revealing the degradation of forests due to cut stumps; ecological factors like forest fires are predominant hence we can conclude that some parts of the study area were under frequent fires, which is degradation to the vegetation.

\section{Conclusion}

The findings of the current study suggested that the species richness ranges from 47-9 in a transect. Species richness was more than 47 for $65 \mathrm{~N} / 14$ (SW-3, 65N/14 NW-3) at Haddubanghi and has least species diversity of 9 at Korasanda 74B/1 (SE-1). Further research should focus on the diversity of the tree species from nearby Srikakulam forest area which will be beneficial to the ecological and taxonomical status of the plant species.

\section{Conflict of Interests}

The author(s) have not declared any conflict of interests.

\section{ACKNOWLEDGEMENT}

The authors are grateful to Department of Biotechnology (DBT) for giving financial assistance and also thank divisional forest officers, forest range officers, guards and beat officers for their help during the field survey.

\section{REFERENCES}

Champion HG, Seth SK (1968). Revised Survey of Forest Types of India. Oxford \& IBH Publishers. New Delhi.

Chandra skhara UM, Sankar S (1998). Ecology and management of sacred groves in Kerala, India. For. Ecol. Manage, 112:165-177.

Chitti Babu CV, Parthasarathy N (2000). Attenuated tree species diversityin human-impacted tropical evergreen forest sites at Kollihills, Estern Ghats, India. Biod. Cons. 9:1493-1519.
Gentry AH (1992). Tropical forest biodiversity: Distributional patterns and their conservational significance. Oikos, 63:19-28.

Holdridge LR (1967). Life Zone Ecology. San Jose, Costa Rica: Tropical Science Center.

Janzen DH (1988). Tropical Dry Forests: The Most Endangered Major Tropical Ecosystem. Wilson, E.O. (Ed), Blod. Nat. Acad. Press, Washington DC., pp. 130-137.

Jayakumar R, Nair KKN (2013). Species diversity and Tree regeneration patterns in Tropical Forests of the Western Ghats, India ISRN Ecology Volume 2013(2013). http://dx.doi.org/10.1155/2013/890862

Jefferies M (1997). Biodiversity and Conservation London \& NewYark

Kadavul K, Parthasarathy N (1998). Biodiversity of woody species and conservation of tropical semi-evergreen forest in the Kalrayan hills of Eastern Ghats, India.

Kadavul K, Parthasarathy N (1999a). Plant biodiversity and conservation of tropical semi-evergreen forest in the Shervarayan hills of Eastern Ghats, India, Biodiv. Cons. 8:421-439.

Kadavul K, Parthasarathy N (1999b). Structure and composition of woody species in tropical semi-evergreen forest of Kalrayan hills, Eastern Ghats, India. Trop. Ecol. 40:247-260.

Mani S, Parthasarathy N (2006). Tree diversity and stand structure in inland and Coastal tropical dry evergreen forests of Peninsular India. Current Science, 90(9):1238-1246.

Murphy PG, Lugo AE (1986). Ecology of tropical dry forests. Ann. Rev. Ecol. Syst. 17:67-88.

Parthasarathy N, Karthikeyan R (1997a). Biodiversity and population density of woody species in a tropical evergreen forest in Courtallum reserve forest, Western Ghats, India. Trop. Ecol. 38:297-306.

Parthasarathy N, Sethi P (1997). Tree and liana species diversity and population structure in a tropical dry evergreen forest in South India. Trop. Ecol. 38:19-30.

Pullaiah T (1997). Flora of Andhra Pradesh, Vol.3. Scientific Publishers, New Delhi.

Pullaiah T, Ali Moulali D (1997). Flora of Andhra Pradesh, Vol. 2. Scientific Publishers Jodhpur.

Pullaiah T, Chennaiah E (1997). Flora of Andhra Pradesh. Vol.1. Scientific Publishers Jodhpur.

Rao RS, Hara Sreeramulu S (1986). The Flora of Srikakulam District. Andhra Pradesh, India, Meerut

Sahu SC, Dhal NK, Sudhakar Reddy, Chiranjibi Pattanaik C, Brahman M (2007). Phytosociological Study of Tropical Dry Deciduous Forest of Boudh District, Orissa, India. Res. J. For. 1(2):66-72.

Srinivasa Rao D, Prayaga Murty P, Aniel Kumar O Srinivasa Rao D et. al (2012). Phytosociological studies on Tree diversity of Srikakulam and Vizianagaram Districts of Andhra Pradesh, India Ph.D. Thesis, Andhra University.

Sudhakar Reddy C, Shilpa Babar, Giriraj A, Reddy KN, Thulsi Rao (2008). Structure and Floristic Composition of Tree Diversity in Tropical Dry Deciduous Forest of Eastern Ghats, Southern Andhra Pradesh, India. Asian J. Sci. Res. 1:57-64.

Sudhakar Reddy P, Murthy MSR, Sudhakar S (2008). Quantitative structure and composition of tropical forests of Mudumalai Wildlife Sanctuary, Western Ghats, India. Taiwania, 53(2):150-156.

Venkaiah M (1980). Studies on the vegetation and flora of

Vizianagaram district, Ph.D. Thesis, Andhra University, Visakhapatnam. 